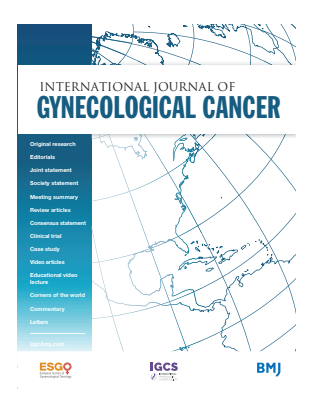

${ }^{1}$ Obstetrics and Gynecology, University of Southern California, Los Angeles, California, USA

${ }^{2}$ Obstetrics and Gynecology, University of Freiburg, Freiburg, Germany

\section{Correspondence to} Dr Koji Matsuo, Obstetrics and Gynecology, University of Southern California, Los Angeles, CA 90033, USA; koji. matsuo@gmail.com

Accepted 25 June 2020 Published Online First 2 August 2020

\title{
Financial viewpoint of minimally invasive radical hysterectomy for early cervical cancer: another disadvantage?
}

\author{
Koji Matsuo (D) ,' Shinya Matsuzaki, ${ }^{1}$ Rachel S Mandelbaum, ${ }^{1}$ Maximilian Klar, ${ }^{2}$ Lynda Roman ${ }^{1}$
}

A battery of analyses of the LACC trial has now added additional information that minimally invasive radical hysterectomy for early cervical cancer is not associated with improved quality of life after surgery compared with open radical hysterectomy. ${ }^{1-3}$ Taken together with the prior principal findings of the LACC trial including (1) increased risk of disease recurrence/death and (2) similar peri-operative morbidity in minimally invasive versus open radical hysterectomy, ${ }^{23}$ the study team recommended that gynecologic oncologists perform open radical hysterectomy for women with early cervical cancer. ${ }^{1}$ Because comparable survival, reduced surgical adverse events, and improved quality of life were the expected premise for performance of minimally invasive over open surgery, failure to demonstrate these three outcome measures argues against the utilization of a minimally invasive approach for cervical cancer treatment.

Another pertinent outcome metric to assess the comparative effectiveness of minimally invasive radical hysterectomy may be a financial one. A recent United States population-based study reported that while length of hospital stay for radical hysterectomy performed for early cervical cancer is shorter with a minimally invasive versus an open approach (1.9 vs 4.3 days), the inpatient cost for surgical and post-operative treatment is higher with robotic-assisted radical hysterectomy compared with open radical hysterectomy (robotic-assisted \$14 029, traditional laparoscopic $\$ 12873$, and open $\$ 12$ 624). ${ }^{4}$ The cost was even higher when the minimally invasive radical hysterectomy was coverted to open (\$17 036). Because robotic-assisted radical hysterectomy represents the majority of minimally invasive radical hysterectomies, ${ }^{5}$ higher treatment cost related to this treatment approach may result in a financial burden as a whole.

Altogether, given the existing data on these four aspects (survival, peri-operative complications, quality of life, and financial considerations), it is difficult to conclude that minimally invasive radical hysterectomy is of significant benefit in the treatment of early cervical cancer. Decreased survival outcomes with minimally invasive radical hysterectomy, which were demonstrated in a recent meta-analysis of 15 studies, are particularly concerning. ${ }^{6}$ As endorsed by the study team, it is paramount to be aware that open radical hysterectomy is the gold standard surgical treatment for women with early cervical cancer.

Contributors KM designed the study, initiated the collaborations, and drafted and revised the manuscript with others; he is also the corresponding author. RSM, SM, and MK contributed to the literature overview, provided intellectual input, interpreted the results, and edited the manuscript. LR supervised the study, and revised the manuscript.

Funding Ensign Endowment for Gynecologic Cancer Research (KM).

Competing interests LR: consultant, Quantgene. KM: honorarium, Chugai; textbook editorial expenses, Springer; and investigator meeting attendance expenses, VBL Therapeutics. SM: research funding, MSD. MK: advisory board, Tesaro, GSK.

Patient consent for publication Not required.

Provenance and peer review Commissioned; internally peer reviewed.

ORCID iD

Koji Matsuo http://orcid.org/0000-0002-6232-8701

\section{REFERENCES}

1 Frumovitz M, Obermair A, Coleman RL, et al. Quality of life in patients with cervical cancer after open versus minimally invasive radical hysterectomy (LACC): a secondary outcome of a multicentre, randomised, open-label, phase 3, non-inferiority trial. Lancet Oncol 2020;21:851-60.

2 Ramirez PT, Frumovitz M, Pareja R, et al. Minimally invasive versus abdominal radical hysterectomy for cervical cancer. N Engl J Med 2018;379:1895-904.

3 Obermair A, Asher R, Pareja R, et al. Incidence of adverse events in minimally invasive vs open radical hysterectomy in early cervical cancer: results of a randomized controlled trial. Am J Obstet Gynecol 2020;222:249.e1-249.e10.

4 Uppal S, Rebecca Liu J, Kevin Reynolds R, et al. Trends and comparative effectiveness of inpatient radical hysterectomy for cervical cancer in the United States (2012-2015). Gynecol Oncol 2019;152:133-8.

5 Matsuo K, Matsuzaki S, Mandelbaum RS, et al. Minimally invasive radical hysterectomy for earlystage cervical cancer: volume-outcome relationship in the early experience period. Gynecol Oncol 2020. doi:10.1016/j.ygyno.2020.05.009. [Epub ahead of print: 27 May 2020].

6 Nitecki R, Ramirez PT, Frumovitz M, et al. Survival after minimally invasive vs open radical hysterectomy for early-stage cervical cancer: a systematic review and meta-analysis. JAMA Oncol 2020. doi:10.1001/ jamaoncol.2020.1694. [Epub ahead of print: 11 Jun 2020]. 